\title{
Rift Valley fever virus-infected mosquito ova and associated pathology: possible implications for endemic maintenance
}

This article was published in the following Dove Press journal:

Research and Reports in Tropical Medicine

16 September 2011

Number of times this article has been viewed

\author{
William S Romoser' \\ Marco Neira Oviedo' \\ Kriangkrai Lerdthusnee ${ }^{2}$ \\ Lisa A Patrican ${ }^{3}$ \\ Michael J Turell ${ }^{4}$ \\ David J Dohm ${ }^{4}$ \\ Kenneth J Linthicum ${ }^{5}$ \\ Charles L Bailey ${ }^{6}$ \\ 'Department of Biomedical Sciences, \\ College of Osteopathic Medicine, \\ Tropical Disease Institute, Ohio \\ University, Athens, Ohio, USA; \\ ${ }^{2}$ Department of Entomology, Faculty \\ of Agriculture, Kasetsart University, \\ Bangkok, Thailand; ${ }^{3}$ Infectious Disease \\ Division, National Center for Medical \\ Intelligence, Fort Detrick, Frederick, \\ Maryland, USA; ${ }^{4}$ Department of \\ Vector Assessment, Virology Division, \\ United States Army Medical Research \\ Institute of Infectious Diseases, Fort \\ Detrick, Frederick, Maryland, USA; \\ ${ }^{5}$ Center for Medical, Agricultural, \\ and Veterinary Entomology, \\ United States Department of \\ Agriculture - Agricultural Research \\ Service, Gainesville, Florida, USA; \\ ${ }^{6}$ National Center for Biodefense and \\ Infectious Disease, School of Systems \\ Biology, College of Science, George \\ Mason University, Manassas, \\ Virginia, USA
}

Correspondence: William S Romoser Tropical Disease Institute, Biomedical Sciences, OUCOM, Ohio University,

Athens, OH 4570I, USA

$\mathrm{Tel}+\mathrm{I} 6145932372$

Fax +l 6145930300

Email wromoser@gmail.com
Background: Endemic/Enzootic maintenance mechanisms like vertical transmission (pathogen passage from infected adults to their offspring) are central in the epidemiology of zoonotic pathogens. In Kenya, Rift Valley fever virus (RVFV) may be maintained by vertical transmission in ground-pool mosquitoes such as Aedes mcintoshi. RVFV can cause serious morbidity and mortality in humans and livestock. Past epidemics/epizootics have occurred in sub-Saharan Africa but, since the late 1970s, RVFV has also appeared in North Africa and the Middle East. Preliminary results revealed RVFV-infected eggs in Ae. mcintoshi after virus injection into the hemocoel after the first of two blood meals, justifying further study.

Methods: Mosquitoes were collected from an artificially flooded water-catching depression along a stream in Kenya, shipped live to the USA, and studied using an immunocytochemical method for RVFV-antigen localization in mosquito sections.

Results and conclusion: After virus injection into the hemocoel, RVFV-infected reproductive tissues were found, particularly follicular epithelia and oocyte/nurse cells. Ovarian infection from the hemocoel is a crucial step in establishing a vertically transmitting mosquito line. Ovarian follicles originate from germarial cells, primordia located distally in each ovariole, and infection of these cells is expected to be requisite for long-term vertical transmission. However, no germarial cell infection was found, so establishing a new line of vertically transmitting mosquitoes may require two generations. The findings support the hypothesis that Ae. mcintoshi is involved in the endemic maintenance of RVFV by vertical transmission. Detection of distinct pathology in infected eggs raises the possibility of virus-laden eggs being deposited among healthy eggs, thereby providing an exogenous source of infection via ingestion by mosquito larvae and other organisms. This has potentially significant epidemiological implications. Possible modes of entry of virus from the hemocoel into the ovaries and routes by which larvae might become infected by ingesting virus are discussed.

Keywords: hemocoel, ovary, vertical transmission, Aedes mcintoshi, arbovirus, antigen positive

\section{Introduction}

The mechanisms of endemic/enzootic maintenance are central to understanding the epidemiology of zoonotic pathogens. An example among arthropod-borne disease systems is vertical transmission, the passage of a pathogen from infected adults to their eggs (transovarial transmission), through the larval instars (transstadial transmission), and, ultimately, to the next generation of adults. Among arthropods, transovarial/ transstadial pathogen transmission was first discovered in ticks relative to the rickettsia associated with Rocky Mountain spotted fever ${ }^{1}$ and later in mosquitoes relative to LaCrosse virus. ${ }^{2}$ Vertical transmission has subsequently been found in several mosquito/arbovirus combinations. ${ }^{3-8}$ submit your manuscript $\mid$ www.dovepress.com
Dovepress

http://dx.doi.org// 0.2147/RRTM.SI3947 
In this paper, evidence for Rift Valley fever virus (RVFV)infected eggs (ova) in the African floodwater mosquito Aedes mcintosh $i^{9}$ after intrathoracic (IT) infection between two blood meals is reported. This finding is consistent with the vertical (transovarial) transmission of RVFV by this species.

Several mosquito-borne arboviruses are active in the USA and, given the introduction and rapid spread of West Nile virus beginning in $1999,{ }^{10,11}$ it is clear that other arboviruses are likewise capable of importation into the USA, for example RVFV. ${ }^{12,13}$ RVFV (genus Phlebovirus, family Bunyaviridae) can cause serious human and livestock morbidity and mortality and is listed as a Category A potential bioterrorism agent. ${ }^{14}$ In the past, RVFV epidemics/epizootics have occurred sporadically throughout much of sub-Saharan Africa, but, from the late 1970s, this virus has appeared in North Africa and the Middle East. ${ }^{15-20}$ Cases associated with a recent outbreak in South Africa continue to appear in 2011. ${ }^{21}$ In sub-Saharan Africa, RVFV is thought to be maintained by vertical transmission in ground-pool Aedes species; for example, one of the hypothetical interepizootic maintenance species in Kenya is the African floodwater mosquito, Ae. mcintoshi. ${ }^{22}$

The authors of this paper have been involved for many years in studies designed to elucidate the interaction between mosquitoes and arboviruses, including the use of in situ methods to locate RVFV antigen and genome segments in sections of mosquito tissues. ${ }^{23-27}$ In a preliminary study of a Kenyan strain of RVFV in Ae. mcintoshi, a single RVFV antigen-positive chorionated egg was found after a second blood meal in a female that had been intrahemocoelically infected after the first blood meal. It was therefore evident that in this species, the reproductive system, particularly the follicular epithelia and oocyte/nurse cells, can become infected with virus originating in the hemocoel. Ovarian infection from the hemocoel after dissemination of virus from the midgut after a viremic blood meal is logically a critical first step in the establishment of a vertically transmitting line of mosquitoes de novo in a previously nonvertically transmitting strain or species. On this basis, the sequence of events that led to infection of follicular epithelia and of an egg was duplicated in a larger sample of mosquitoes.

While the research reported here was carried out several years ago, the ideas presented have developed from discussions, especially between the first and second authors during a study of western equine encephalitis virus infection in Culex tarsalis in which the same phenomenon was observed. The study of western equine encephalitis virus/
Cx. tarsalis will be reported separately, but the results are fully consistent with our findings in RVFV-infected Ae. mcintoshi.

\section{Materials and methods Mosquito collection}

Specimens of Ae. mcintoshi were collected from an artificially flooded dambo (natural water-catching depression along a stream) at Sakari Ranch near Nairobi, Kenya, and shipped live to the US Army Medical Research Institute of Infectious Diseases (USAMRIID), Fort Detrick, Frederick, Maryland.

\section{Mosquito infection and analysis}

In a preliminary study at USAMRIID, four mosquitoes that had obtained blood from an unknown host in Kenya were inoculated with RVFV (Kenya strain 21445) by injection into the hemocoel through a soft area of cuticle in the thorax (IT infection/innoculation), held at $26^{\circ} \mathrm{C}$ for several days, allowed to oviposit, and then given a second blood meal from an uninfected hamster. These mosquitoes were killed and fixed for immunocytochemical examination after being held long enough for vitellogenesis to occur.

Based on preliminary results, a larger sample of adult female mosquitoes that had been collected in Kenya and held at USAMRIID were likewise IT inoculated with the 21445 strain of RVFV. After 3 days incubation, mosquitoes were allowed to ingest, digest, and assimilate a blood meal from an uninfected hamster and provided with an opportunity to oviposit. A second blood meal opportunity was then provided and the resulting 35 engorged females incubated for about 3 days. Gravid females were then killed and fixed in 5\% formaldehyde for approximately 5 hours and stored in $70 \%$ ethyl alcohol. IT-inoculated mosquitoes and similarly treated uninfected virus-diluent-injected female Culex pipiens mosquitoes (100\% infection rate when IT inoculated) were prepared as positive and negative controls, respectively.

\section{Immunocytochemistry}

To detect viral antigen in serial paraffin sections of mosquitoes, the avidin-biotin-peroxidase complex immunocytochemical technique for light-level microscopy was used..$^{28,29}$ The "primary antibody" was a blend of monoclonal antibodies directed against RVFV nucleocapsid protein and two envelope glycoproteins or a monoclonal antibody directed against RVFV nucleocapsid protein, both effective in antigen detection. Dr James Meegan and Dr Jonathan Smith provided 
these antibodies. Appropriate reagent elimination studies had been carried out earlier. ${ }^{29}$

\section{Results}

In the preliminary study, one of the four specimens had an antigen-positive egg with a distinct positive signal in all serial sections of that egg as well as an antigen-positive follicular epithelium. The results of the 35 RVFV-infected female Ae. mcintoshi study are shown in Table 1 and Figure $1 \mathrm{~A}-\mathrm{H}$. Representative sections from control specimens are shown in Figure 1I-L.

Among the ultimate follicles with chorionated eggs, RVFV antigen was seen in a single egg in three different specimens; ie, $3 / 35$ or $8.6 \%$. Antigen could be seen in several serial sections of each infected egg along with distinct pathology characterized by the coalescence of yolk granules having a somewhat clumped, smooth appearance (Figure 1A and B). This pathology was in contrast to the granular yolk texture observed in uninfected eggs adjacent to the infected eggs (Figure 1A and B) and in the control (Figure 1L).

Both ovarian (Figure 1E) and ovariole sheaths (Figure 1A and D) were observed to contain antigen in many individuals, $74.3 \%$ and $51.4 \%$, respectively. In no case was the germarium (Figure 1B) observed to contain antigen. A single penultimate follicle was observed to be antigen positive. A much higher frequency of antigen presence was observed in the follicular epithelia of ultimate follicles (Figure 1A and C), 62.9\% in the cells associated with the anterior (distal) part of an egg,

Table I RVFV antigen in Aedes mcintoshi female reproductive tissues

\begin{tabular}{ll}
\hline Tissue/Structure & N RVFV Antigen + I \\
& N observed (\% Ag +) \\
\hline Ovarian sheath & $26 / 35(74.3)$ \\
Ovariole sheath & $18 / 35(5 I .4)$ \\
Germarium & $0 / 35(0)$ \\
Penultimate follicle: & \\
Epithelium & $1 / 35(2.9)$ \\
Oocyte/Nurse cells & $1 / 35(2.9)$ \\
Ultimate follicle: & \\
Oocyte/Nurse cells & $3 / 35(8.6)$ \\
Epithelium-anterior & $22 / 35(62.9)$ \\
Epitehelium-central & $19 / 34(55.9)$ \\
Epithelium-posterior (pedicel/calyx) & $27 / 32(84.4)$ \\
Lateral oviduct & $32 / 35(91.4)$ \\
Common oviduct & $31 / 34(91.2)$ \\
Genital chamber & $26 / 33(78.8)$ \\
Cells near spermathecal pores & $20 / 31(64.5)$ \\
Spermatozoa & $0 / 23(0)$ \\
Accessory gland & $0 / 25(0)$ \\
\hline Abbreviations Ag &
\end{tabular}

Abbreviations: $\mathrm{Ag}+$, antigen positive; $\mathrm{N}$, number; RVFV, Rift Valley fever virus.
$55.9 \%$ in the cells of the central region of an egg, and $84.4 \%$ in the cells associated with the posterior (proximal) region of an egg (including the adjacent pedicel and calyx). The oviducts (Figure 1F) displayed high frequencies of antigen presence, as did cells near the spermathecae (Figure $1 \mathrm{~F}$ and $\mathrm{H}$ ). In no case was the accessory gland (Figure $1 \mathrm{H}$ ), or spermatozoa, when present in the spermathecae, seen to contain antigen. Many instances of antigen in the trachea associated with the ovaries were observed (Figure 1G). In one case a chorionated egg contained distinct traces of RVFV antigen (Figure 1C).

\section{Discussion}

The findings of this study are consistent with the hypothesis that Ae. mcintoshi is involved in the endemic maintenance of RVFV by vertical transmission. ${ }^{22}$ The occurrence of RVFV antigen-positive eggs is evidence that RVFV in Ae. mcintoshi can reach and infect eggs from the hemocoel when there is a disseminated infection (virus in the hemocoel beyond the midgut epithelium). The fact that mosquitoes were IT inoculated, given an uninfected blood meal, allowed to oviposit, and then given a second blood meal may have influenced the receptivity of the ovaries to RVFV infection.

The occurrence of strongly antigen-positive eggs with antigen in virtually every section, along with associated pathology, suggests that eggs heavily infected with RVFV can be deposited in the aquatic habitat along with uninfected viable eggs. The observation of distinct traces of antigen in an otherwise normal-appearing egg (Figure 1C) suggests that some eggs could have a mild or even latent viral infection with no pathology and which might remain viable.

The appearance of distinct antigen in the cytoplasm of smaller tracheae suggests that virus entry into the ovaries could possibly occur via ovarian tracheae. It has been suggested that tracheae could be virus escape conduits from the midgut epithelium into the hemolymph (dissemination). ${ }^{25,26}$ Virus could enter the ovaries by direct infection of the ovarian and ovariolar sheaths and then the follicular epithelia. Because antigen was observed in all of these tissues, it is possible that both tracheal and sheath routes are involved in infection of the ovaries.

Regardless of mode of ingress into an ovary, infection of a developing egg with virus from the hemocoel can logically be expected to be a critical step in the establishment de novo of a line of vertically transmitting mosquitoes. A permanent vertically transmitting line of mosquitoes would ultimately require infection of the germaria, the undifferentiated 


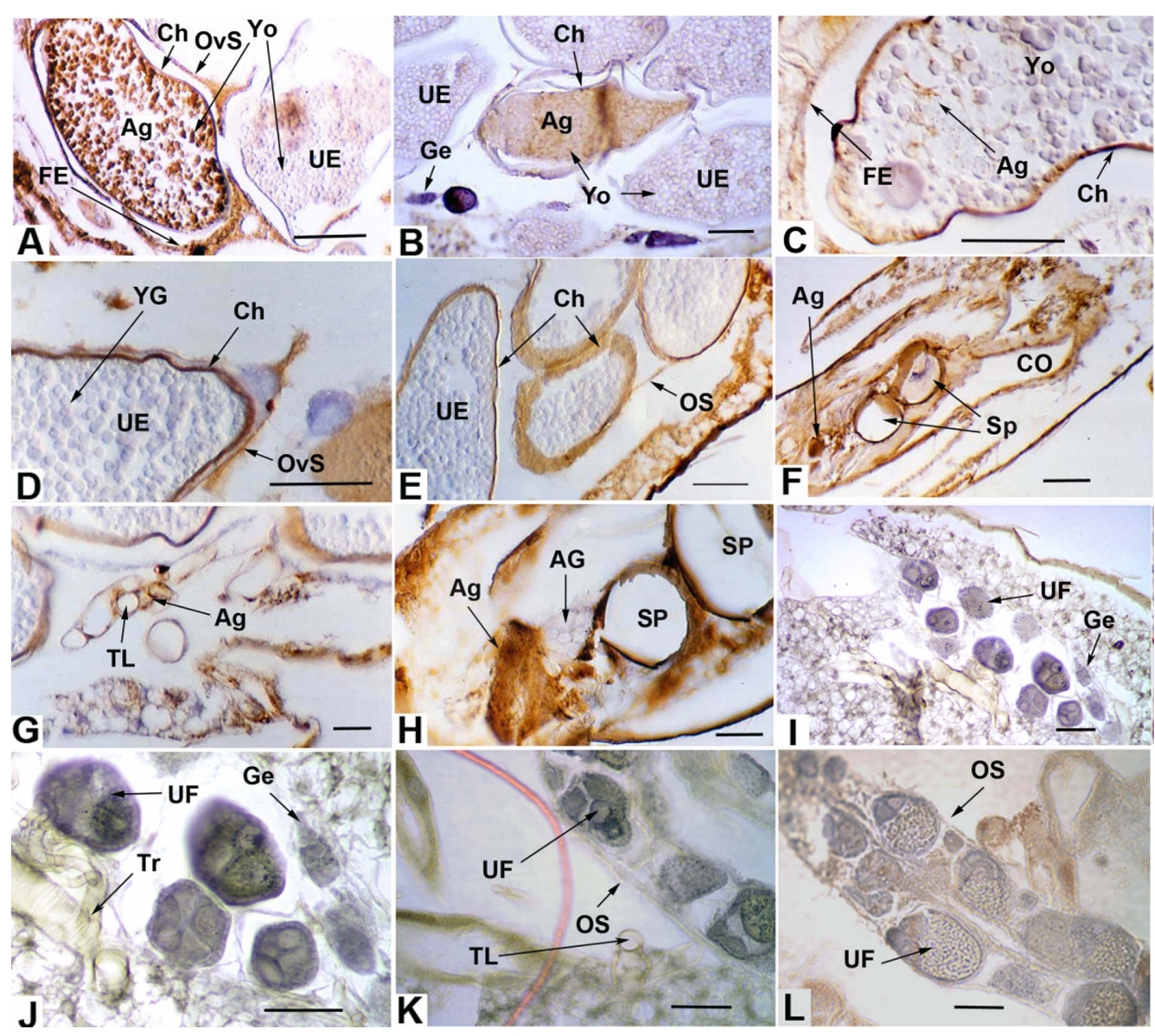

Figure I (A, B) RVFV antigen in chorionated eggs showing pathology and RVFV antigen-negative germarium (B). (C) Chorionated egg with distinct traces of RVFV antigen. (D) Uninfected chorionated egg with antigen-positive ovariolar sheath. (E) Uninfected chorionated eggs with RVFV antigen-positive ovarian sheath. (F) Sagittal section in posterior part of the abdomen showing antigen-positive oviduct epithelium and tissues in proximity to the spermathecae. (G) Antigen-positive small tracheae associated with the ovaries. (B) Section of spermathecae and adjacent antigen-positive tissues, and antigen-negative accessory gland. (I-L) Negative control sections.

Note: Scale lines $(\mu \mathrm{m})$ : A-E, I50; F, I00; G, 20; H, 50; I-L, 50.

Abbreviations: Ag, RVFV antigen; AG, accessory gland; Ch, chorion; CO, common oviduct; FE, follicular epithelium; Ge, germarium; OS, ovary sheath; OvS, ovariole sheath; RVFV, Rift Valley fever virus; Sp, spermatheca; TL, tracheal lumen; Tr, trachea; UE, uninfected egg; UF, uninfected follicle; Yo, yolk.

primordial tissue at the apex of each ovariole from which the oocytes/nurse cells and follicular epithelia originate. Virus in germarial cells would have ample opportunity to infect oocytes and follicular epithelia as they develop. It seems safe to assume that many, if not all, follicles originating from an infected germarium would be infected. However, no evidence of antigen in germarial tissue was observed. How, then, could a permanent vertically transmitting line be established?

The finding of what appears to be a lightly infected egg suggests the possibility of other eggs having undetected or latent infections. If such eggs remained viable, a line of a vertically transmitting mosquito generations with a "stabilized" viral infection ${ }^{30}$ could be established. This is because infection would be systemic from the very beginning as opposed to the virus from a viremic blood meal being required to infect several differentiated tissues to spread. Virus that has infected an egg would have opportunity to spread during virtually all of the processes of growth and development, that is, during embryogenesis, during the larval stadia, and during metamorphosis to the adult stage. Virus present during metamorphosis would be in a position to infect any developing adult tissues, including primordial germ cells and adult salivary gland cells. Infected female germ cells would be expected to produce at least some infected germaria and hence ovarioles that would produce infected eggs. Any infected but viable eggs among all eggs deposited would be expected to undergo the same sequence of tissue-infection events during growth and development, thereby leading to infected eggs in the next generation, and so on, a generational repeat of vertical transmission, that is, a stabilized infection. ${ }^{30}$ 
Likewise, such a systemic infection in a male egg might produce infected male gametes (spermatozoa), raising the possibility of venereal transmission, a phenomenon first described in male Aedes triseriatus, tree-hole mosquitoes infected with LaCrosse encephalitis virus ${ }^{31}$ and later in two other mosquito-arbovirus combinations. ${ }^{32,33}$ Infection of spermatheca-associated tissues could lead to "transovum" transmission, in which case virus might eventually enter chorionated eggs via the micropyle along with spermatozoa as they enter eggs passing along the oviduct.

Though admittedly speculative, another way a permanent vertically transmitting line could be established stems from the fact that heavily infected eggs displayed signs of pathology. This indicates that likely nonviable virus-laden eggs can be deposited along with viable uninfected eggs. Once in the aquatic environment, these eggs could conceivably be a source of viral infection for mosquito larvae or other aquatic organisms. A logical route of egress of virus from an infected egg would be via the micropyle, the opening through which sperm enter and then initiate fertilization. The eventual breakdown of a heavily infected egg could also release virus. Larvae from viable eggs deposited along with one or more infected eggs and larvae from female adults of the same or different species could easily ingest released virus particles. Infected eggs (or larvae) are also likely to be ingested by other animals (vertebrate or invertebrate), offering a putative mechanism for cross-specific viral transmission. ${ }^{34}$ Such transmission within the larval habitat could play a very significant role in endemic maintenance and could also enhance virus spread and amplification associated with animal and/or human disease outbreaks. Mosquito larval habitats generally teem with other organisms, including arthropods and other invertebrates, Monera, and Protists, ${ }^{35}$ and it is well known that various vertebrates (fish, tadpoles, frogs, toads, birds, and bats) prey on mosquito larvae and/or adults.

The idea of infected eggs releasing virus, which infect larvae when ingested, is consistent with the demonstration that larval ingestion of particles from homogenized, RVFV-infected hamster livers in the rearing medium lead to transstadial virus transmission and produce adults that transmit virus to new hosts during blood feeding. ${ }^{36}$ It is unclear how infected hamster livers and infected mosquito eggs compare in regard to the amount of virus present, but the very strong positive reaction of the egg shown in Figure 1A is indicative of widespread infection within the egg and suggests the possibility that the viral titer within an infected egg could be reasonably high.
To establish infection in a mosquito larva, ingested virus has to come into contact with midgut epithelial cell surface-receptor molecules associated with the plasma membranes. However, the larval foregut is lined with an impermeable, noncellular chitinous intima ${ }^{37,38}$ and ingested particles in the midgut lumen are completely invested in the cylindrical noncellular peritrophic membrane (PM). The larval PM, while permeable to large molecules such as the mosquitoes' own proteolytic enzymes, ${ }^{39,40}$ is not permeable to virus-sized particles. However, the work of Turell et al ${ }^{36}$ demonstrates that ingested RVFV particles must in fact reach plasma membrane and infect epithelial cells. The question remains regarding how virus-sized particles circumvent the foregut intima and larval PM and initiate infection. Relative to the structure of the mosquito alimentary canal and to developmental changes that happen in the fourth instar larval stage, there are at least two possibilities. First, it seems possible that virus could reach the foregut-midgut junction within the larval proventriculus, immediately anterior to where the larval PM forms but posterior to the cells to which the foregut intima attaches and terminates. There is evidence that RVFV can infect the corresponding region in adult mosquitoes and possibly disseminate from there into the hemocoel. ${ }^{23,27} \mathrm{~A}$ second plausible way for ingested virus to reach the plasma membranes of larval midgut epithelial cells is during the pharate pupal stage at the time when the larval PM is destroyed or egested but before the meconial PM forms. ${ }^{41}$ In this regard, it is noteworthy that Turell et al used fourth instar larvae in their experiments. ${ }^{36}$

Although four individual eggs infected in four different mosquitoes may seem to be a low frequency of infection, that 4/35 individuals (11.4\%) contain any RVFV antigenpositive eggs could be very significant, especially in view of the potentially large populations of floodwater mosquitoes emerging in a short period of time. Arbovirus-infected egg pathology has also been observed in $C x$. tarsalis in association with an arbovirus in a different family, that is, the Togaviridae, ${ }^{42}$ suggesting that our observations of infected eggs associated with apparent pathology are not just random artifacts and may well play a role in the establishment of a line of vertically transmitting mosquitoes.

\section{Disclosure}

This research was financially supported by the US Army Research and Development Command (contract number DAMD17-86-C-6133). 
The views of the authors do not purport to reflect the positions of the US Department of the Army or the US Department of Defense. Research was conducted in compliance with the Animal Welfare Act and other federal statutes and regulations relating to animals and experiments involving animals and adheres to principles stated in the Guide for the Care and Use of Laboratory Animals, National Research Council, 1996. The facility where this research was conducted is fully accredited by the Association for Assessment and Accreditation of Laboratory Animal Care International.

\section{References}

1. Burgdorfer W. Vertical transmission of spotted fever group and scrub typhus rickettsiae. In: Harris KF, editor. Current Topics in Vector Research. Santa Barbara, CA: Praeger; 1984:77-92.

2. Watts DM, Pantuwatana S, Yuill TM, DeFoliart GR, Thompson WH, Hanson RP. Transovarial transmission of LaCrosse virus in Aedes triseriatus. Ann N Y Acad Sci. 1975;266:135-143.

3. Leake CJ. Transovarial transmission of arboviruses by mosquitoes. In: Mayo MA, Harrap KA, editors. Vectors in Virus Biology. London: Academic Press; 1984:63-91.

4. Rosen L. Ovarian infection and transovarial transmission of viruses in insects. In: Notkins AL, Oldstone MBA, editors. Concepts in Viral Pathogenesis. New York, NY: Springer-Verlag; 1984:194-198.

5. Tesh RB. Transovarial transmission of arboviruses in their invertebrate vectors. In: Harris KF, editor. Current Topics in Vector Research. Santa Barbara, CA: Praeger; 1984:57-76.

6. Turell MJ. Horizontal and vertical transmission of viruses by insect and tick vectors. In: Monath TP, editor. The Arboviruses: Epidemiology and Ecology. Boca Raton, FL: CRC Press; 1988:128-152.

7. Wilson ML. Rift Valley fever virus ecology and the epidemiology of disease emergence. Ann N Y Acad Sci. 1994;740:169-180.

8. Higgs S. Influences of arthropod vectors on encephalic arboviruses. In: Shoskes Reiss C, editor. Neurotropic Viral Infections. Cambridge, UK: Cambridge University Press; 2008:362-381.

9. Huang Y-M. A new African species of Aedes (Diptera: Culicidae). Mosquito Systematics. 1985;17:108-120. Available from: http://www. mosquitocatalog.org/files/pdfs/MS17N02P108.pdf. Accessed August 18, 2011.

10. Calisher CH. West Nile virus in the New World: appearance, persistence, and adaptation to a new econiche - an opportunity taken. Viral Immunol. 2000;13(4):411-414.

11. Hayes EB, Komar N, Nasci RS, Montgomery SP, O'Leary DR, Campbell GL. Epidemiology and transmission dynamics of West Nile virus disease. Emerg Infect Dis. 2005;11(8):1167-1173.

12. Gargan TP 2nd, Clark GG, Dohm DJ, Turell MJ, Bailey CL. Vector potential of selected North American mosquito species for Rift Valley fever virus. Am J Trop Med Hyg. 1988;38(2):440-446.

13. Turell MJ, Dohm DJ, Mores CN, et al. Potential for North American mosquitoes to transmit Rift Valley fever virus. J Am Mosq Control Assoc. 2008;24(4):502-507.

14. Centers for Disease Control and Prevention. Bioterrorism Agents/ Diseases. Atlanta, GA: Centers for Disease Control and Prevention; 2011. Available from: http://emergency.cdc.gov/agent/agentlistcategory.asp. Accessed July 20, 2011.

15. Arthur RR, el-Sharkawy MS, Cope SE, et al. Recurrence of Rift Valley fever in Egypt. Lancet. 1993;342(8880):1149-1150.

16. Meegan JM, Bailey CL. Rift Valley fever. In: Monath TP, editor. Arboviruses: Epidemiology and Ecology. Boca Raton, FL: CRC Press; 1989;51-76.
17. Meegan JM. The Rift Valley fever epizootic in Egypt 1977-1978. Description of the virological studies. Trans R Soc Trop Med Hyg. 1979;73(6):618-623.

18. Meegan JM, Khalil GM, Hoogstraal H, Adham FK. Experimental transmission and field isolation studies implicating Culex pipiens as vector of Rift Valley fever in Egypt. Am J Trop Med Hyg. 1980;29(6): 1405-1410.

19. Fagbo SF. The evolving transmission pattern of Rift Valley fever in the Arabian Peninsula. Ann NY Acad Sci. 2002;969:201-204.

20. Abdo-Salem S, Gerbier G, Bonnet P, et al. Descriptive and spatial epidemiology of Rift valley fever outbreak in Yemen 2000-2001. Ann NY Acad Sci. 2006;1081:240-242.

21. The Outbreak Response Unit, Special Pathogens Unit, and South African Field Epidemiology and Laboratory Training Programme (SAFELTP), National Institute for Communicable Diseases (NICD) of the National Health Laboratory Service (NHLS). Rift Valley Fever Outbreak: Interim Report on the 2011 Rift Valley Fever (RFV) Outbreak in South Africa. 2011 [update Mar 22]. Available from: http://www. samedical.org/assets/files/doctors-corner/outbreaks/rift-valleyfever/Rift $\% 20$ Valley\%20Fever\%20Outbreak\%2022\%20March.pdf. Accessed July 20, 2011.

22. Linthicum KJ, Davies FG, Kairo A, Bailey CL. Rift Valley fever virus (family Bunyaviridae, genus Phlebovirus). Isolations from Diptera collected during an inter-epizootic period in Kenya. J Hyg (Lond). 1985;95(1):197-209.

23. Romoser WS, Faran ME, Bailey CL. A newly recognized route of arbovirus dissemination from the mosquito midgut. J Med Entomol. 1987;24(4):431-432.

24. Romoser WS, Faran ME, Bailey CL, Lerdthusnee K. An immunocytochemical study of the distribution of Rift Valley fever virus in the mosquito Culex pipiens. Am J Trop Med Hyg. 1992;46(4): 489-501.

25. Romoser WS, Wasieloski LP Jr, Pushko P, et al. Evidence for arbovirus dissemination conduits from the mosquito midgut. $J$ Med Entomol. 2004;41(3):467-475.

26. Romoser WS, Turell MJ, Lerdthusnee K, et al. Pathogenesis of Rift Valley fever virus in mosquitoes - tracheal conduits and the basal lamina as an extra-cellular barrier. Arch Virol Suppl. 2005;19:89-100.

27. Lerdthusnee K, Romoser WS, Faran ME, Dohm DJ. Rift Valley fever virus in the cardia of Culex pipiens: an immunocytochemical and ultrastructural study. Am J Trop Med Hyg. 1995;53(4):331-337.

28. Hsu SM, Raine L, Fanger H. Use of avidin-biotin-peroxidase complex $(\mathrm{ABC})$ in immunoperoxidase techniques: a comparison between $\mathrm{ABC}$ and unlabeled antibody (PAP) procedures. J Histochem Cytochem. 1981;29(4):577-580.

29. Faran ME, Romoser WS, Routier RG, Bailey CL. Use of the avidinbiotin-peroxidase complex immunocytochemical procedure for detection of Rift Valley fever virus in paraffin sections of mosquitoes. Am J Trop Med Hyg. 1986;35(5):1061-1067.

30. Turell MJ, Hardy JL, Reeves WC. Stabilized infection of California encephalitis virus in Aedes dorsalis, and its implications for viral maintenance in nature. Am J Trop Med Hyg. 1982;31(6):1252-1259.

31. Thompson WH, Beaty BJ. Venereal transmission of La Crosse (California encephalitis) arbovirus in Aedes triseriatus mosquitoes. Science. 1977;196(4289):530-531.

32. Shroyer DA. Venereal transmission of St. Louis encephalitis virus by Culex quinquefasciatus males (Diptera: Culidicae). J Med Entomol. 1990;27(3):334-337.

33. Mavale M, Parashar D, Sudeep A, et al. Venereal transmission of chikungunya virus by Aedes aegypti mosquitoes (Diptera: Culicidae). Am J Trop Med Hyg. 2010;83(6):1242-1244.

34. Sbrana E, Tonry JH, Xiao SY, da Rosa AP, Higgs S, Tesh RB. Oral transmission of West Nile virus in a hamster model. Am J Trop Med Hyg. 2005;72(3):325-329.

35. Laird M. The Natural History of Larval Mosquito Habitats. Oxford: Academic Press; 1988. 
36. Turell MJ, Linthicum KJ, Beaman JR. Transmission of Rift Valley fever virus by adult mosquitoes after ingestion of virus as larvae. Am J Trop Med Hyg. 1990;43(6):677-680.

37. Romoser WS, Venard CE. The development of the ventral oesophageal diverticulurn in Aedes triseriatus (Diptera: Culicidae). Ann Entomol Soc Am. 1967;59(3):484-489.

38. Walker MC, Romoser WS. The origin and movement of gas during adult emergence in Aedes aegypti: an hypothesis. J Am Mosq Control Assoc. 1987;3(3):429-432.

39. Detra RL, Romoser WS. Permeability of Aedes aegypti (L.) (Diptera: Culicidae) larval peritrophic membrane to proteolytic enzyme. Mosquito News. 1979;39(3):582-585.

40. Edwards MJ, Jacobs-Lorena M. Permeability and disruption of the peritrophic matrix and caecal membrane from Aedes aegypti and Anopheles gambiae mosquito larvae. J Insect Physiol. 2000;46(9): 1313-1320.
41. Moncayo AC, Lerdthusnee K, Leon R, Robich RM, Romoser WS. Meconial peritrophic membrane structure, formation and meconial degeneration in mosquito pupae/pharate adults: histological and ultrastructural aspects. J Med Entomol. 2005;42(6):939-944.

42. Neira Oviedo MV, Romoser WS, James CBL, Mahmood F, Reisen WK. Infection dynamics of western equine encephalomyelitis virus (Togaviridae: Alphavirus) in four strains of Culex tarsalis (Diptera: Culicidae): an immunocytochemical study. Res Rep Trop Med. 2011; 2011(2):65-77.

\section{Publish your work in this journal}

Research and Reports in Tropical Medicine is an international, peerreviewed, open access journal publishing original research, case reports, editorials, reviews and commentaries on all areas of tropical medicine, including: Diseases and medicine in tropical regions; Entomology; Epidemiology; Health economics issues; Infectious disease; Laboratory science and new technology in tropical medicine; Parasitology; Public health medicine/health care policy in tropical regions; and Microbiology. The manuscript management system is completely online and includes a very quick and fair peer-review system. Visit http://www.dovepress. com/testimonials.php to read real quotes from published authors.

Submit your manuscript here: http://www.dovepress.com/research-and-reports-in-tropical-medicine-journal 